\title{
Comparison of Echocardiographic parameters with Cardiac Magnetic Resonance Imaging in the Assessment of Right Ventricular Function
}

Pradyumna Agasthi ${ }^{1}$, Chieh-Ju Chao, ${ }^{1}$, Robert J. Siegel ${ }^{2}$, Sai Harika Pujari ${ }^{1}$, Farouk Mookadam $^{3}$, Nithin Venepally ${ }^{1}$, Panwen Wang ${ }^{1}$, Hasan Ashraf ${ }^{1}$, Francois Marcotte ${ }^{1}$, Lisa Brown $^{1}$, Oksana Semkiv ${ }^{1}$, Ayman Fath ${ }^{1}$, Mohamed Allam ${ }^{1}$, Clinton Jokerst ${ }^{1}$, and Reza Arsanjani $^{1}$

${ }^{1}$ Mayo Clinic Arizona

${ }^{2}$ Cedars Sinai Med Ctr

${ }^{3}$ Mayo Clinic

June 10, 2020

\begin{abstract}
Introduction: The right ventricle (RV) strain measured by speckle tracking (RVS) is a novel method of assessing RV function. We compared RVS to RV fractional area change (FAC\%), tricuspid annular peak systolic excursion (TAPSE) and Doppler tissue imaging-derived peak systolic velocity (S') in the assessment of right ventricular (RV) systolic function measured using cardiac magnetic resonance imaging (MRI). Methods: We enrolled consecutive patients who underwent cardiac MRI between Jan 2012- Dec 2017 and a transthoracic echocardiogram (TTE) within 1 month of the MRI with no interval event. Baseline clinical characteristics and MRI parameters were extracted from chart review. Echocardiographic parameters were measured prospectively. TTE parameters including RVS, TAPSE, S' and FAC\% were tested for accuracy to identify impaired RV EF (EF $<45 \% \&<30 \%)$ using receiver operator curves. Results: The study cohort included 500 patients with mean age 55 yr \pm 18 and right ventricular systolic pressure $33.7 \pm 13.6 \mathrm{mmHg}$. The area under ROC for RVS was 0.69 (95\% CI $0.63-0.75)$ and $0.78(95 \%$ CI $0.70-0.88)$ to predict RVEF $<45 \%$ \& RVEF $<30 \%$ respectively. The RV FAC $\%$ had second highest accuracy of predicting RVEF among all the TTE parameters tested in study. Conclusion: Right ventricular strain is the most accurate echocardiographic method to detect impaired right ventricular systolic function when using MRI as the gold standard.
\end{abstract}

Comparison of Echocardiographic parameters with Cardiac Magnetic Resonance Imaging in the Assessment of Right Ventricular Function

\section{Authors:}

Pradyumna Agasthi M.D ${ }^{a}$; Chieh-Ju Chao, M.D ${ }^{a}$, Robert J. Siegel M.D ${ }^{\text {, }}$, Sai Harika Pujari M.B.B.S ${ }^{\text {a }}$, Farouk Mookadam M.B.B.Ch ${ }^{\mathrm{a}}$, Nithin R. Venepally M.B.B.S a , Panwen Wang PhD ${ }^{\mathrm{c}}$, Hasan Ashraf M.D ${ }^{\mathrm{a}}$, Francois Marcotte M.D ${ }^{a}$, Lisa Brown R.D.C.S ${ }^{a}$, Oksana I. Semkiv R.D.C.S ${ }^{a}$, Ayman R. Fath M.B.Ch.B ${ }^{\text {a }}$, Mohamed N.A. Allam M.D ${ }^{a}$, Clinton E. Jokerst M.D ${ }^{d}$, Reza Arsanjani M.D ${ }^{a}$

a) Department of Cardiovascular Diseases, Mayo Clinic Arizona, Phoenix, Arizona

b) Department of Cardiology, Cedars Sinai Medical Center, Los Angeles, California

c) Department of Radiology, Mayo Clinic Arizona, Phoenix, Arizona

d) Department of Health Sciences Research, Mayo Clinic Arizona, Scottsdale, Arizona 
Disclosure: The authors report no financial relationships or conflicts of interest regarding the content herein.

Corresponding Author: Pradyumna Agasthi M.D

Institutional Affiliation: Mayo Clinic Arizona

Address: 5777 East Mayo Boulevard, Phoenix, AZ 85054

Telephone: 7864938374

Email: pradyumna_agasthi@hotmail.com

\section{Structured Abstract:}

Introduction:

The right ventricle (RV) strain measured by speckle tracking (RVS) is a novel method of assessing RV function. We compared RVS to RV fractional area change (FAC\%), tricuspid annular peak systolic excursion (TAPSE) and Doppler tissue imaging-derived peak systolic velocity (S') in the assessment of right ventricular (RV) systolic function measured using cardiac magnetic resonance imaging (MRI).

Methods:

We enrolled consecutive patients who underwent cardiac MRI between Jan 2012- Dec 2017 and a transthoracic echocardiogram (TTE) within 1 month of the MRI with no interval event. Baseline clinical characteristics and MRI parameters were extracted from chart review. Echocardiographic parameters were measured prospectively. TTE parameters including RVS, TAPSE, S' and FAC\% were tested for accuracy to identify impaired RV EF (EF $<45 \%$ \& <30\%) using receiver operator curves.

Results:

The study cohort included 500 patients with mean age $55 \mathrm{yr} \pm 18$ and right ventricular systolic pressure $33.7 \pm 13.6 \mathrm{mmHg}$. The area under ROC for RVS was $0.69(95 \%$ CI $0.63-0.75)$ and 0.78 (95\% CI $0.70-$ $0.88)$ to predict RVEF $<45 \%$ \& RVEF $<30 \%$ respectively. The RV FAC $\%$ had second highest accuracy of predicting RVEF among all the TTE parameters tested in study.

Conclusion:

Right ventricular strain is the most accurate echocardiographic method to detect impaired right ventricular systolic function when using MRI as the gold standard.

Key Words: 1) right ventricular function 2) Echocardiography 3) Cardiac magnetic resonance imaging 4) Strain imaging

\section{Introduction:}

Right Ventricular (RV) function has significant prognostic importance for a variety of heart diseases and their clinical outcomes. RV function assessment is frequently conducted for staging and prognostic purposes, especially in cardiac diseases with a right heart involvement. Of note, RV dysfunction can affect the left ventricular (LV) Forward stroke volume by restricting the preload of LV, as well as alter LV systolic and diastolic function secondary to the interaction with the interventricular septum and pericardium, and eventually lead to biventricular failure. This is ascribed to the physiological function of RV to maintain adequate pulmonary perfusion pressure and a low systemic venous pressure.

Current clinical management has largely relied on non-invasive modalities like echocardiography for RV function assessment. Conventionally, one-dimensional echocardiography parameters such as tricuspid annular peak systolic excursion (TAPSE), lateral tricuspid annular systolic velocity (S') and two-dimensional (2D) parameters such as RV functional area change (FAC) are being used. However, these parameters are still subject to limitations due to the complex anatomical structure and geometric shape of RV. The RV rendering volume readings by echocardiography are susceptible to errors due to the distinctive cavity geometry of the 
RV as opposed to the ellipsoid shape of LV, and the change in longitudinal contraction pattern in diseased states.

Cardiac magnetic resonance imaging (CMR) is considered as the gold standard for RV function assessment. CMR provides accurate results in quantifying heart function and structure and has become widely used for different heart diseases. In RV assessment, RV volumes obtained by CMR indicate a strong correlation with clinical assessment, and there is an excellent accuracy and consistency of the volume measurements. Despite the high accuracy and reproducibility, CMR has its constraints including cost, availability, time-consuming, and skill-consuming as it requires drawing endocardial contour manually, and also requires defining the most basal short-axis slice and the endocardial border, which is complicated by thin free wall and extensive trabeculations .

With the recent advance of speckle-tracking echocardiography, direct measurement of myocardial deformation by strain becomes a great alternative tool for RV function assessment. RV strain has been reported as a technique with high accuracy and reproducibility and is closely correlated with prognosis. However, only limited studies have compared all these parameters with CMR. The purpose of this study is to evaluate the accuracy of echocardiographic parameters in assessing RV systolic function and specifically to compare standard echo parameters to RV strain imaging and MRI to identify the best echo parameter(s) to assess RV systolic function

\section{Methods:}

\section{Study individuals:}

We enrolled consecutive patients who underwent CMR between Jan 2012- Dec 2017 and a transthoracic echocardiogram (TTE) within 1 month of the CMR with no interval clinical event. The study cohort included five hundred subjects at Mayo Clinic Arizona. The following Doppler echocardiographic parameters for assessment of RV systolic function: Tricuspid annular peak systolic excursion (TAPSE), lateral tricuspid annular systolic velocity (S'), lateral RV free wall peak systolic strain (S), and RV fractional area change (RV FAC), and cardiac MRI (RVEF) were acquired in all the subjects. Subdivisions in the patient group are based on RVEF measured by CMR: RVEF [?] 45\% ( $\mathrm{n}=333$, Group A), RVEF 30-45\% ( $\mathrm{n}=140$, Group B), and RVEF [?] 30\% ( $\mathrm{n}=27$, Group C). All echocardiographic parameters were reassessed by the investigators for the current study.

\section{Doppler echocardiography:}

Standard ultrasound scanners (Philips iE33; Philips Medical Systems, Andover, MA, USA; GE Vivid E9, GE Healthcare, Milwaukee, WI, USA) with a $3.5 \mathrm{MHz}$ transducer, including tissue Doppler imaging (TDI) was used for transthoracic Doppler echocardiography and strain echocardiography was conducted using a speckle tracking software. Both conventional M-mode and 2D echocardiography were performed from the left parasternal and apical window. Each measurement was taken carefully after examining patients in both supine and left lateral positions. During the end-systole and end-diastole, M-mode LV readings were acquired. LV EF was calculated with LV volume measurements taken in biplane projection from apical twochamber and four-chamber views. Using the end-diastolic values of septal and posterior wall thickness and LV cavity dimension, LV mass was determined as per the cube formula. Bi-apical Simpson rule was applied to compute LV volumes and the simplified Bernoulli equation to calculate the tricuspid pressure gradient.

\section{Right Ventricular Dimensions:}

Two-dimensional echocardiography was used to derive RV area and length measurements during end-systole and end-diastole from the apical four-chamber view, and RV fractional area change was assessed. Final figures were produced after an average of three cardiac cycles. Lateral TAPSE was computed as the distance amid the tricuspid annulus greatest apical long-axis movement and its basal, end-diastolic position taken at the beginning of ECG QRS complex and was determined by M-mode echocardiography. A 2-4 mm sampling gate and a $200 \mathrm{~mm} / \mathrm{s}$ sweep were used to attain pulsed-wave TDI of the peak systolic tricuspid annular motion velocity at the lateral free wall $\left(\mathrm{S}^{\prime}, \mathrm{cm} / \mathrm{s}\right)$ in apical four-chamber view . 


\section{Speckle-tracking strain analysis:}

Speckle tracking strain echocardiography was used to determine RV lateral free wall average peak systolic strain . RV strain was measured using ECHOINSIGHT software (version 2.2.6.2230, Epsilon Imaging, Ann Arbor, MI). Based on the 4-chamber apical view, three successive RV cardiac cycles with a frame rate of 70120/s were recorded. Right ventricular endocardial border was manually traced in still end-systolic frames, and the software performed automated tracking throughout the cardiac cycle. The automated tracking was visually verified and manually adjusted for adequate border delineation. Tissue velocities in the 2D plane are displayed throughout the cardiac cycle, representing magnitude and direction of motion. Finally, strain data was generated by analyzing the speckle pattern along the endocardial border in reference to one another. Longitudinal strain is defined as the percentage shortening of a regional area of interest (ROI) relative to its original length and is expressed as a negative value. The software further categorized the RV myocardium into six sections: basal RV free wall, mid-RV free wall, apical free wall, left basal septum, mid-left septum, and left apical septum and calculated the regional wall strain with the same manner. RV lateral free wall strain (average of the basal, mid, and apical free wall) was used in our analysis. Figure 1 demonstrated a representative case of RV dimension measurement.

\section{Reproducibility:}

Reproducibility was assessed using 50 randomly chosen individuals from all the three subgroups. The four echocardiographic parameters and RVEF (assessed by MRI) were measured by two blinded observers (to both echocardiography and MRI data), and inter-observer variability was evaluated. The standard error of estimate of the mean percent signifies measurement reproducibility.

\section{Cardiac magnetic resonance imaging:}

Periodic changes of the cardiac chambers and ventricular walls were visualized and interpreted by an experienced observer blinded to echocardiographic findings and clinical information. The imaging was performed in the supine position on all subjects using a $1.5 \mathrm{~T}$ (Magnetom Symphony) or a $3 \mathrm{~T}$ (Magnetom Trio, both from Siemens Medical Solutions, Erlangen, Germany) whole-body clinical MRI system. All images were tracked for end-systolic and end-diastolic endocardial contours. Modified Simpson's rule was used to determine RV volumes. Three electrodes positioned on the left anterior hemithorax and cardiac synchronization was acquired. Commercially accessible (Argus version 4.01, Syngo MR B 13, Siemens Medical Solutions, Erlangen, Germany) software accomplished an evaluation of images. A mid-ventricular axial view, a cine breath-hold vertical long axis, and a cine breath-hold horizontal long axis established cardiac short axis. During short end-expiratory breath-holds, the ventricles were imaged in $8 \mathrm{~mm}$ increments using adjacent short-axis slices from the base to apex while the basal short-axis slice was placed beyond the mitral valve plane. Long axis views verified the short-axis acquisitions for comprehensive coverage of ventricles.

\section{Statistical analysis:}

Descriptive statistics were summarized as mean +- standard deviation or number with percentage. Statistically relevant correlations between the Doppler echocardiographic parameters and RVEF by CMR were achieved by linear regression analysis. Receiver operating characteristics (ROC) curves were obtained by analyzing the variables of echocardiography as the sample factors and RVEF by CMR as the state variable. Shapiro-Wilk test was made use to determine the normal distribution of data, whereas an analysis of variance (ANOVA) was used to compare amongst the groups of Doppler echocardiographic variables and other demographic variables. As mentioned above, the standard error of estimate of the mean percent signifies inter-observer variability. We used R 3.4.1 (Foundation for Statistical Computing, Vienna, Austria) to run our analysis.

\section{Results:}

Patient Characteristics:

When the three groups are compared, the patients did not differ regarding age, body mass index, or height. 
A higher percentage of men were present in patients with impaired RVEF. Hypertension was predominant in the patients with normal RVEF, and diabetes mellitus was more common in the patients with an impaired RVEF. As identified in table 1, cardiovascular diagnoses varied among the groups, but cardiomyopathy remained the most frequent diagnosis in all patients.

\section{Echocardiographic Variables:}

Table 2 shows the echocardiographic variables of the subgroups. The right ventricular systolic pressure was $30.97+/-9.27,36.72+/-14.50$, and $48.12+/-13.92$, respectively, in normal, moderately reduced, and severely reduced EF patients $(\mathrm{p}<0.0001)$. Reproducibility of inter-observer variabilities was calculated for all the four variables, S', TAPSE, RV FAC, and lateral RV free wall strain. There was a significant difference in the parameters assessed for RV systolic function (Table 2). There was a linear association with the MRI reference parameter of RVEF with RVEF $<45 \%$ and RVEF $<30 \%$ for all the variables, with strain having the most significant association (Figure $\mathbf{2}$ and Figure 3 ). Other parameters like LV mass index, LV end-diastolic mass, and LV end-diastolic mass indices also significantly differed among the groups. ROC curves with cutoffs of RVEF $<45 \%$, and RVEF [?] 30\% are shown in Figure 4 estimating the accuracy of echocardiographic parameters to predict RV function. The echocardiographic parameter that most accurately assessed the RV systolic function was RV lateral free wall strain and the area under the curve (AUC) for ROC was $0.69(95 \%$ CI $0.63-0.75)$ and 0.78 (95\% CI $0.70-0.88)$ in the groups B and C respectively (Table 3) . RV FAC \% ranked second in the accuracy of predicting RV systolic function among the TTE parameters in both groups.

\section{Reproducibility}

The standard error values of each parameter are $3.74 \%\left(\mathrm{r}^{2}=0.5605\right), 3.68 \%\left(\mathrm{r}^{2}=0.389\right), 4.09 \%\left(\mathrm{r}^{2}=0.619\right)$, $5.024 \%\left(\mathrm{r}^{2}=0.889\right)$ and $2.24 \%\left(\mathrm{r}^{2}=0.922\right)$, for $\mathrm{S}$, TAPSE, RV FAC, lateral RV free wall strain and MRI derived RVEF respectively.

\section{Discussion:}

In our study comprising 500 consecutive patients with a broad spectrum of cardiovascular disorders, we evaluated the accuracy of commonly used echocardiographic parameters, including peak systolic annular velocity (s'), tricuspid annular plane systolic excursion (TAPSE), RV fractional area change (RV FAC), as well as RV strain, compared to CMR RV ejection fraction (RVEF) in assessing RV systolic function. To the best of our knowledge, this is the largest study that contains a variety of patient population and compares echocardiographic parameters with RVEF measured by CMR. This is also the first study to demonstrate the superior accuracy of RV free wall strain compared to other echocardiographic parameters to distinguish RVEF $<45 \%$ and RVEF $<30 \%$.

Similar to previous studies, we choose CMR measured RV ejection fraction (RVEF) as our reference standard for RV systolic function assessment. CMR enjoys the advantage of 3D volumetric information and high spatial resolution to analyze the distinctive geometry of RV, which is usually difficult to obtain by using $2 \mathrm{D}$ echocardiography measurements. The accuracy and reproducibility of using CMR for quantitative RV assessment have been demonstrated in multiple clinical studies. As a functional parameter, RVEF reflects the contractility of right heart myocardium, and can be affected by RV preload, afterload, and other general variables including age, sex and body mass index. Age and gender did not differ significantly between the groups in our study, but the percentage of men was higher in Group B.

Among the four echocardiographic parameters, our results indicate that RV strain and RV FAC are in general superior to TAPSE and S' in accuracy, when compared with CMR RVEF by both spearman's correlation and ROC curve analyses. Our results demonstrated a comparable or better reproducibility of each parameter, compared to prior studies. This finding suggests that two-dimensional parameters are superior two onedimensional parameters and implies the importance of using two-dimensional or even three-dimensional tools for accurate RV function assessment concerning its complex geometry.

$R V$ peak systolic annular velocity $\left(S^{\prime}\right)$ 
As a conventional RV function parameter, $\mathbf{S}$ ' represents the tricuspid annulus' longitudinal velocity at systole and is easy to estimate, reliable and reproducible. Previous studies show that S' might be the best predictor of RVEF, and may predict short-term outcome of heart failure. S' was also questioned whether the displacement of the myocardium or the regional velocities in a single segment can truly represent the entire RV function. However, our observation indicates the accuracy of $S^{\prime}$ 'in assessing RV systolic dysfunction maybe not as good as suggested in prior studies . Using a cutoff of $<10 \mathrm{~cm} / \mathrm{s}$, the accuracy (AUC) of $\mathrm{S}^{\prime}$ in differentiating the severity of RV systolic dysfunction were in the range of $0.58-0.65$ in our study; which is apparently lower than previously reported 0.7-0.8 range. Of note, the S' threshold of RV systolic dysfunction varies considerably between studies while the commonly accepted S' cutoff for RV dysfunction is $<10 \mathrm{~cm} / \mathrm{s}$. In addition, a study showed that S' detected best at a cut-off value of $8.8 \mathrm{~cm} / \mathrm{sec}$ in 10 patients with arrhythmogenic RV dysplasia and low RVEF. Another study found that S' between lateral and septal tricuspid annulus of $7 \mathrm{~cm} / \mathrm{s}$ was the most accurate means to detect an RVEF cut-off by MRI of $45 \%$. Overall, our data support that $\mathrm{S}^{\prime}$ is more accurate in cases with mild RV dysfunction as reported previously. The S' cutoff used in prior studies ranged from $7 \mathrm{~cm} / \mathrm{s}$ to $11 \mathrm{~cm} / \mathrm{s}$, which is about 30 to $40 \%$ variation between different studies. This implies a single best cutoff threshold may not exist among different patient cohorts.

Tricuspid annulus peak systolic excursion (TAPSE)

TAPSE had similar performance as S' in our study and was slightly more accurate than S' in patients with severe RV dysfunction $\left(\mathrm{AUC}_{\mathrm{TAPSE}} 0.673>\mathrm{AUC}_{\mathrm{S}}, 0.652\right)$. TAPSE is also readily obtainable, and has modest correlation with RVEF, 2D RV FAC and 2D RV EF although it measures the longitudinal function. TAPSE is a simple and straightforward measurement that doesn't require optimal image quality and time-consuming image analysis. Similar to S', TAPSE assumes that the basal and adjacent segment displacement in the fourchamber view represents the entire RV function, which may not be valid in several disease states and regional RV wall motion abnormalities. In other words, TAPSE is comparatively load- and angle dependent, can subject to cardiac translation and is the least user dependent parameter for RV function assessment.

\section{RV FAC}

In our study, the accuracy of RV FAC was comparable with RV free wall strain in patients with mild and moderate RV dysfunction; the AUC range was from 0.62 to 0.69. RV FAC was superior to the 1-dimensional parameters, S' and TAPSE. As a well-established 2-dimensional parameter, RV FAC has been validated in various studies and was shown to be an independent indicator of sudden death, stroke, and heart failure. A recent study indicated that RV FAC can accurately predict RVEF than $45 \%$, with an AUC of 0.89. Potential limitations of RV FAC include lack of clear guidance in selecting the optimal view for measurements of a 3-dimensional object with complex geometric shape, and RV FAC neglects contribution of RVOT to the overall RV function.

\section{Speckle-tracking $R V$ free wall strain}

RV lateral free wall strain had the best accuracy among the four echocardiographic parameters. It consistently outperformed other parameters in identifying different levels of RV systolic dysfunction and was most accurate with severe RV systolic dysfunction. We identified cutoff levels of lateral RV free wall strain of $<23.5 \%$ and $<20.5 \%$ that accurately detects for moderately and severely reduced RV systolic function, respectively. Although the RV free wall strain technically measures more of the longitudinal deformation of the RV myocardium, it has been validated as a global function parameter in a variety of diseases with promising results and predicting prognosis. Compared to LV, base-to-apex shortening plays a more important role in RV systolic function due to its distinct muscle fiber architecture. This also partially explained the superiority of longitudinal RV strain in assessing RV systolic function. In addition, speckle-tracking RV strain also has the unique advantage of being angle-independent, and able to measure the intrinsic myocardium deformation. An issue of using RV strain is the different reference values generated by the software package from different vendors; one should be cautious when comparing RV strain data from different studies. The ease of performance for sonographers comparing all four modalities in descending order are TAPSE, S', FAC and RV strain. Theoretically, RV myocardial strain should be superior to localized velocity measurements 
such as S'. Our study demonstrates strain is superior to other echo parameters as compared with Cardiac MRI as the standard.

In conclusion, conventional one-dimensional echocardiographic parameters, such as S' and TAPSE, were well-validated and have the advantage of simplicity, reproducibility and are less dependent on image quality. However, either TAPSE or S' in our study were not as good as reported in prior RV dysfunction studies. The 2-dimensional parameters, RV FAC and RV free wall strain were superior in accuracy. The ease of performance for sonographers comparing all four modalities in descending order are TAPSE, S', FAC and RV strain.

Considering the availability, reproducibility, accuracy and the correlation to prognosis, our results suggested that RV free wall strain is the preferred choice for RV functional assessment among the four echocardiographic parameters.

As a final comment, the AUC of all four parameters improved with worsening RV dysfunction, which reflects the challenge in detecting mild to moderate RV dysfunction in clinical practice. Despite the relatively good AUC data, all the four echocardiographic parameters only had modest correlations with CMR RVEF. This implied a substantial limitation on 1- or 2-dimensional assessment of RV systolic function. In clinical practice, these parameters can serve as surrogates of RV dysfunction concerning their availability and relatively low costs. However, when accurate assessment of RV systolic function is warranted, 3-dimensional tools like cardiac MRI should be the preferred choice when it's available.

\section{Study limitations:}

Our study aims to provide a general picture of RV dysfunction assessments by different echocardiographic parameters in different diseases, so a disease-specific analysis was not performed. Statistically, the ROC analysis showing the accuracy gives a satisfactory correlation. However, the inherent limitations of measurement of variables exist. The study did not use three-dimensional echocardiography, and the right ventricle's unique geometry makes it difficult to obtain images using two-dimensional planes. RVEF is a function of both longitudinal and concentric myocardial fibers, while tricuspid annular systolic motion principally indicates the longitudinal fibers' function only. The angle of interrogation and cardiac motion influence the velocity measurement, especially from the apical four-chamber view during long axis assessment limiting the accuracy of S'. As mentioned above, RVEF measured by MRI can account for some unknown extent of variability of data. Lack of 3D echocardiographic assessment of RV function in our dataset remains a limitation in our study. Prior studies have shown a good correlation 3D TTE with MRI for the assessment of RV function. Good 3D assessment of RV function requires excellent image quality, lack of arrhythmia and long breath holds during image acquisition. It is prone to stich artifacts, needs cumbersome software processing, has low frame rate and significant inter-vendor variability, hampering routine use of 3D RV assessment in contemporary practice.

\section{Conclusion:}

In conclusion, our study is the largest study to date to compare a multiparameter echo-Doppler assessment of RV systolic function with RVEF determined by cardiac MR for validation. Our results suggest the superiority of lateral RV free wall strain to other conventional echocardiographic parameters for detecting RV systolic dysfunction. Lateral RV free wall strain of $<23.5 \%$ accurately detects moderately reduced RVEF and lateral RV free wall strain $<20.5 \%$ accurately detects severely reduced RV systolic function.

Conflict of interest/Disclosures: None of the authors have any disclosures. Informed, written consent was obtained from all individuals for whom information is included in this article. The authors have conformed to institutional guidelines and those of the American Physiological Society.

\section{Funding :}

This research did not receive any specific grant from funding agencies in the public, commercial, or not-forprofit sectors. 


\section{Table Legends:}

Table 1: Patient Characteristics.

Table 2: Baseline Echocardiographic and Cardiac Magnetic Resonance Data

Table 3: Area under the Receiver Operating Curve Characteristics of Echocardiographic parameters to predict right ventricular ejection fraction measured using cardiac magnetic resonance imaging.

\section{Figure Legends:}

Figure 1: A representative case of RV dimension measurements. A) TAPSE B) RV peak myocardial contraction velocity (S'), C) End-diastole and D) End-systole phases of RV fractional area change, respectively, E) speckled tracking strain measurements of RV.

Figure 2: Spearman Correlation graphs in the study patients with RVEF <45\%: Right ventricular (RV) ejection fraction measured by cardiac magnetic resonance imaging (CMR) on vertical axis and Doppler echocardiographic variables on horizontal axes. A) Lateral tricuspid annular peak systolic velocity measured by tissue Doppler imaging, S' B) Lateral RV free wall peak systolic strain (\%) C) RV fractional long axis change (\%), RV FAC D) Tricuspid annular peak systolic excursion, TAPSE (mm).

Figure 3: Spearman Correlation graphs in the study patients with RVEF $<30 \%$ : Right ventricular (RV) ejection fraction measured by cardiac magnetic resonance imaging (CMR) on vertical axis and Doppler echocardiographic variables on horizontal axes. A) Lateral tricuspid annular peak systolic velocity measured by tissue Doppler imaging, S' B) Lateral RV free wall peak systolic strain (\%) C) RV fractional long axis change (\%), RV FAC D) Tricuspid annular peak systolic excursion, TAPSE (mm).

Figure 4: Receiver operating characteristic analysis curves for the distinction by the echocardiographic parameters measured in the study. A) Right ventricular ejection fraction (RVEF) $<45 \%$ B) RVEF [?] 30\%; RV FAC- RV Fractional long axis change, S'- Lateral tricuspid annular peak systolic velocity, TAPSETricuspid annular peak systolic excursion.

CRediT author statement

Pradyumna Agasthi: Conceptualization, Methodology, Data Curation, Investigation, Visualization, WritingOriginal Draft, Writing-Review \&Editing

Chieh-Ju Chao: Conceptualization, Data Curation, Writing-Original Draft, Writing-Review \&Editing

Robert J. Siegel: Supervision, Conceptualization, Methodology, Writing-Review \&Editing

Sai Harika Pujari: Data Curation, Visualization, Writing-Original Draft, Writing-Review \&Editing

Farouk Mookadam: Supervision, Conceptualization, Methodology, Writing-Review \&Editing

Nithin R. Venepally: Data Curation, Visualization, Writing-Review \&Editing

Panwen Wang: Methodology, Investigation, Formal Analysis, Writing-Review \&Editing

Hasan Ashraf: Conceptualization, Data Curation, Writing-Original Draft, Writing-Review \&Editing

Francois Marcotte: Supervision, Conceptualization, Methodology, Writing-Review \&Editing.

Lisa Brown: Data Curation, Visualization, Writing-Review \&Editing

Oksana Semkiv: Data Curation, Visualization, Writing-Review \&Editing

Ayman Fath: Data Curation, Visualization, Writing-Review \&Editing

Mohamed Allam: Data Curation, Visualization, Writing-Review \&Editing

Clinton E. Jokerst: Supervision, Conceptualization, Methodology, Writing-Review \&Editing 
Reza Arsanjani: Supervision, Project Administration, Conceptualization, Methodology, Writing-Review \&Editing

References:

Table 1: Patient Characteristics.

\begin{tabular}{|c|c|c|c|c|}
\hline & $\begin{array}{l}\text { Group A Right } \\
\text { Ventricular } \\
\text { ejection fraction } \\
{[?] \mathbf{4 5 \%}(\mathrm{n}=333)}\end{array}$ & $\begin{array}{l}\text { Group B Right } \\
\text { Ventricular } \\
\text { ejection fraction } \\
>30-45 \% \\
(\mathrm{n}=140)\end{array}$ & $\begin{array}{l}\text { Group C Right } \\
\text { Ventricular } \\
\text { ejection fraction } \\
\text { [?] } \mathbf{3 0 \%}(\mathbf{n}=\mathbf{2 7})\end{array}$ & P-value \\
\hline Age (years) & $56.02 \pm 17.88$ & $55.04 \pm 18.59$ & $54.61 \pm 16.79$ & 0.5404 \\
\hline Men n (\%) & $199(59.76)$ & $94(67.14)$ & $17(62.96)$ & 0.0370 \\
\hline $\begin{array}{l}\text { Body mass } \\
\text { index }\left(\mathrm{kg} / \mathrm{m}^{2}\right)\end{array}$ & $27.26 \pm 5.40$ & $27.06 \pm 4.52$ & $27.34 \pm 4.39$ & 0.8323 \\
\hline Height $(\mathrm{cm})$ & $172.22 \pm 9.57$ & $173.52 \pm 9.11$ & $176.05 \pm 11.38$ & 0.0274 \\
\hline $\begin{array}{l}\text { Diabetes } \\
\text { Mellitus n (\%) }\end{array}$ & $44(13.21)$ & $25(17.86)$ & $0(0)$ & $<0.0001$ \\
\hline $\begin{array}{l}\text { Hypertension } \mathbf{n} \\
(\%)\end{array}$ & $152(45.65)$ & $50(35.71)$ & $0(0)$ & $<0.0001$ \\
\hline $\begin{array}{l}\text { Sleep Apnea n } \\
(\%)\end{array}$ & $65(19.52)$ & $29(20.71)$ & $0(0)$ & $<0.0001$ \\
\hline $\begin{array}{l}\text { Primary } \\
\text { cardiovascular } \\
\text { diagnosis: } \\
\text { Normal n }(\%)\end{array}$ & $\begin{array}{l}\text { Primary } \\
\text { cardiovascular } \\
\text { diagnosis: } \\
94(28.23)\end{array}$ & $\begin{array}{l}\text { Primary } \\
\text { cardiovascular } \\
\text { diagnosis: } \\
24(17.14)\end{array}$ & $\begin{array}{l}\text { Primary } \\
\text { cardiovascular } \\
\text { diagnosis: } \\
(0)\end{array}$ & $\begin{array}{l}\text { Primary } \\
\text { cardiovascular } \\
\text { diagnosis: } \\
<0.0001\end{array}$ \\
\hline $\begin{array}{l}\text { Coronary } \\
\text { artery disease } n \\
(\%)\end{array}$ & $43(12.91)$ & $18(12.86)$ & $6(22.22)$ & $<0.0001$ \\
\hline $\begin{array}{l}\text { Cardiomyopathy } \\
\text { n (\%) }\end{array}$ & $142(42.64)$ & $66(47.14)$ & $24(88.89)$ & $<0.0001$ \\
\hline $\begin{array}{l}\text { Valvular heart } \\
\text { disease } \mathrm{n}(\%)\end{array}$ & $39(11.71)$ & $16(11.43)$ & $4(14.81)$ & $<0.0001$ \\
\hline $\begin{array}{l}\text { Tetralogy of } \\
\text { Fallot } \mathrm{n}(\%)\end{array}$ & $3(0.9)$ & $4(2.86)$ & $1(3.7)$ & $<0.0001$ \\
\hline $\begin{array}{l}\text { Transposition } \\
\text { of great } \\
\text { arteries n (\%) }\end{array}$ & $0(0)$ & $1(0.71)$ & $0(0)$ & $<0.0001$ \\
\hline $\begin{array}{l}\text { Other } \\
\text { congenital } \\
\text { heart disease } \mathbf{n} \\
(\%)\end{array}$ & $11(3.3)$ & $9(6.43)$ & $1(3.7)$ & $<0.0001$ \\
\hline Other n (\%) & $37(11.11)$ & $22(15.71)$ & $3(11.11)$ & $<0.0001$ \\
\hline $\begin{array}{l}\text { Pulmonary } \\
\text { hypertension } n \\
(\%)\end{array}$ & $4(1.2)$ & $9(6.43)$ & $1(3.7)$ & $<0.0001$ \\
\hline
\end{tabular}

Table 2: Baseline Echocardiographic and Cardiac Magnetic Resonance Data 


\begin{tabular}{|c|c|c|c|c|}
\hline & $\begin{array}{l}\text { Group A Right } \\
\text { Ventricular } \\
\text { ejection fraction } \\
{[?] 45 \%(n=333)}\end{array}$ & $\begin{array}{l}\text { Group B Right } \\
\text { Ventricular } \\
\text { ejection fraction } \\
>30-45 \% \\
(\mathrm{n}=140)\end{array}$ & $\begin{array}{l}\text { Group C Right } \\
\text { Ventricular } \\
\text { ejection fraction } \\
\text { [?] } \mathbf{3 0 \%}(\mathrm{n}=\mathbf{2 7})\end{array}$ & P-value \\
\hline $\begin{array}{l}\text { Doppler } \\
\text { echocardio- } \\
\text { graphic } \\
\text { data }\end{array}$ & $\begin{array}{l}\text { Doppler } \\
\text { echocardio- } \\
\text { graphic } \\
\text { data }\end{array}$ & $\begin{array}{l}\text { Doppler } \\
\text { echocardio- } \\
\text { graphic } \\
\text { data }\end{array}$ & $\begin{array}{l}\text { Doppler } \\
\text { echocardio- } \\
\text { graphic } \\
\text { data }\end{array}$ & $\begin{array}{l}\text { Doppler } \\
\text { echocardio- } \\
\text { graphic } \\
\text { data }\end{array}$ \\
\hline $\begin{array}{l}\text { LV mass index } \\
(\mathrm{g}, \mathrm{m} 2)\end{array}$ & $102.45 \pm 34.71$ & $114.25 \pm 45.98$ & $129.54 \pm 42.04$ & $<0.0001$ \\
\hline $\begin{array}{l}\text { Left atrial } \\
\text { volume index } \\
\left(\mathrm{ml} / \mathrm{m}^{2}\right)\end{array}$ & $33.66 \pm 12.50$ & $36.86 \pm 13.84$ & $45.17 \pm 20.54$ & $<0.0001$ \\
\hline $\mathrm{E}(\mathrm{cm} / \mathrm{sec})$ & $0.79 \pm 0.24$ & $0.79 \pm 0.3$ & $0.81 \pm 0.25$ & 0.8041 \\
\hline $\begin{array}{l}\text { e'_medial_- } \\
\text { annulus } \\
(\mathrm{cm} / \mathrm{sec})\end{array}$ & $0.07 \pm 0.03$ & $0.07 \pm 0.03$ & $0.05 \pm 0.03$ & 0.1591 \\
\hline $\begin{array}{l}\text { e'_lateral_annulus } \\
(\mathrm{cm} / \mathrm{sec})\end{array}$ & $0.19 \pm 1.34$ & $0.09 \pm 0.04$ & $0.07 \pm 0.03$ & 0.3427 \\
\hline E/e'_medial & $13.65 \pm 7.79$ & $13.22 \pm 7.14$ & $19.06 \pm 10.15$ & 0.1514 \\
\hline E/e'_lateral & $10.97 \pm 6.29$ & $11.12 \pm 6.68$ & $12.47 \pm 6.69$ & 0.4245 \\
\hline $\begin{array}{l}\text { Right } \\
\text { ventricular } \\
\text { systolic } \\
\text { pressure } \\
(\mathrm{mmHg})\end{array}$ & $30.97 \pm 9.27$ & $36.72 \pm 14.50$ & $48.12 \pm 13.92$ & $<0.0001$ \\
\hline $\begin{array}{l}\text { Left ventricular } \\
\text { ejection } \\
\text { fraction }(\%)\end{array}$ & $60.19 \pm 12.65$ & $50.20 \pm 16.13$ & $28.44 \pm 15.29$ & $<0.0001$ \\
\hline $\begin{array}{l}\text { Left ventricular } \\
\text { end diastolic } \\
\text { diameter }(\mathrm{mm})\end{array}$ & $46.50 \pm 7.22$ & $50.21 \pm 8.05$ & $59.81 \pm 11.08$ & $<0.0001$ \\
\hline $\begin{array}{l}\text { Left ventricular } \\
\text { end systolic } \\
\text { diameter }(\mathrm{mm})\end{array}$ & $30.09 \pm 8.22$ & $36.57 \pm 12.78$ & $49.26 \pm 13.92$ & $<0.0001$ \\
\hline $\begin{array}{l}\text { Right } \\
\text { ventricular } \\
\text { fractional area } \\
\text { change (FAC) } \\
(\%)\end{array}$ & $41.71 \pm 8.70$ & $38.67 \pm 9.63$ & $32.89 \pm 12.23$ & $<0.0001$ \\
\hline $\begin{array}{l}\text { Lateral free } \\
\text { wall peak } \\
\text { systolic strain, } \\
\text { S (\%) }\end{array}$ & $-26.12 \pm 16.10$ & $-26.22 \pm 29.04$ & $-18.30 \pm 6.52$ & 0.2142 \\
\hline $\begin{array}{l}\text { Lateral } \\
\text { tricuspid } \\
\text { annular systolic } \\
\text { velocity, S' } \\
(\mathrm{cm} / \mathrm{sec})\end{array}$ & $10.72 \pm 30.17$ & $8.48 \pm 2.48$ & $7.70 \pm 2.67$ & 0.3181 \\
\hline
\end{tabular}




\begin{tabular}{|c|c|c|c|c|}
\hline & $\begin{array}{l}\text { Group A Right } \\
\text { Ventricular } \\
\text { ejection fraction } \\
{[?] \mathbf{4 5 \%}(\mathrm{n}=\mathbf{3 3 3})}\end{array}$ & $\begin{array}{l}\text { Group B Right } \\
\text { Ventricular } \\
\text { ejection fraction } \\
>30-45 \% \\
(\mathrm{n}=140)\end{array}$ & $\begin{array}{l}\text { Group C Right } \\
\text { Ventricular } \\
\text { ejection fraction } \\
{[?] \mathbf{3 0 \%}(\mathrm{n}=\mathbf{2 7})}\end{array}$ & P-value \\
\hline $\begin{array}{l}\text { Tricuspid } \\
\text { annular plane } \\
\text { systolic } \\
\text { excursion, } \\
\text { TAPSE (mm) }\end{array}$ & $20.84 \pm 5.49$ & $19.93 \pm 6.21$ & $16.11 \pm 8.65$ & 0.0003 \\
\hline $\begin{array}{l}\text { Cardiac MRI } \\
\text { data }\end{array}$ & $\begin{array}{l}\text { Cardiac MRI } \\
\text { data }\end{array}$ & $\begin{array}{l}\text { Cardiac MRI } \\
\text { data }\end{array}$ & $\begin{array}{l}\text { Cardiac MRI } \\
\text { data }\end{array}$ & $\begin{array}{l}\text { Cardiac MRI } \\
\text { data }\end{array}$ \\
\hline $\begin{array}{l}\text { Left ventricular } \\
\text { end diastolic } \\
\text { mass }(\mathrm{g})\end{array}$ & $132.45 \pm 53.19$ & $137.99 \pm 56.97$ & $167.66 \pm 56.46$ & 0.0087 \\
\hline $\begin{array}{l}\text { Left ventricular } \\
\text { end diastolic } \\
\text { mass indices } \\
\left(\mathrm{g} / \mathrm{m}^{2}\right)\end{array}$ & $69.73 \pm 26.80$ & $70.81 \pm 26.97$ & $84.12 \pm 24.61$ & 0.0771 \\
\hline $\begin{array}{l}\text { Right } \\
\text { ventricular end } \\
\text { diastolic } \\
\text { volume }(\mathrm{ml})\end{array}$ & $144.63 \pm 42.54$ & $161.60 \pm 52.55$ & $227.24 \pm 85.81$ & $<0.0001$ \\
\hline $\begin{array}{l}\text { Indexed Right } \\
\text { ventricular end } \\
\text { diastolic } \\
\text { volume }(\mathrm{ml})\end{array}$ & $104.15 \pm 529.43$ & $84.04 \pm 25.88$ & $116.82 \pm 39.26$ & 0.8348 \\
\hline $\begin{array}{l}\text { Right } \\
\text { ventricular end } \\
\text { systolic volume } \\
(\mathrm{ml})\end{array}$ & $59.45 \pm 22.90$ & $93.70 \pm 32.06$ & $175.85 \pm 73.64$ & $<0.0001$ \\
\hline $\begin{array}{l}\text { Indexed Right } \\
\text { ventricular end } \\
\text { systolic volume } \\
(\mathrm{ml})\end{array}$ & $30.77 \pm 11.51$ & $48.22 \pm 16.90$ & $90.82 \pm 34.25$ & $<0.0001$ \\
\hline $\begin{array}{l}\text { Right } \\
\text { ventricular } \\
\text { stroke volume } \\
(\mathrm{ml})\end{array}$ & $85.13 \pm 23.57$ & $69.56 \pm 23.53$ & $51.47 \pm 19.65$ & $<0.0001$ \\
\hline $\begin{array}{l}\text { Indexed Right } \\
\text { ventricular } \\
\text { stroke volume } \\
(\mathrm{ml})\end{array}$ & $43.72 \pm 11.17$ & $35.92 \pm 11.01$ & $25.38 \pm 8.97$ & $<0.0001$ \\
\hline $\begin{array}{l}\text { Right } \\
\text { ventricular } \\
\text { ejection } \\
\text { fraction }(\%)\end{array}$ & $59.36 \pm 6.79$ & $42.79 \pm 5.21$ & $23.45 \pm 6.27$ & $<0.0001$ \\
\hline
\end{tabular}

Table 3: Area under the Receiver Operating Curve Characteristics of Echocardiographic parameters to predict right ventricular ejection fraction measured using cardiac magnetic resonance imaging. 


\begin{tabular}{ll}
\hline & AUC \\
\hline Group B (Right Ventricular Ejection Fraction <45\%) & Group B (Right Ventricular Ejection Fraction $<45$ \\
Strain (RV free wall) (\%) & 0.6866 \\
S' (cm/sec) & 0.5909 \\
FAC (\%) & 0.6658 \\
TAPSE (mm) & 0.5819 \\
Group C (Right Ventricular Ejection Fraction < 30\%) & Group C (Right Ventricular Ejection Fraction $<\mathbf{3}$ \\
Strain (RV free wall) (\%) & 0.7795 \\
S' (cm/sec) & 0.6525 \\
FAC (\%) & 0.6942 \\
TAPSE $(\mathbf{m m})$ & 0.6732 \\
\hline
\end{tabular}




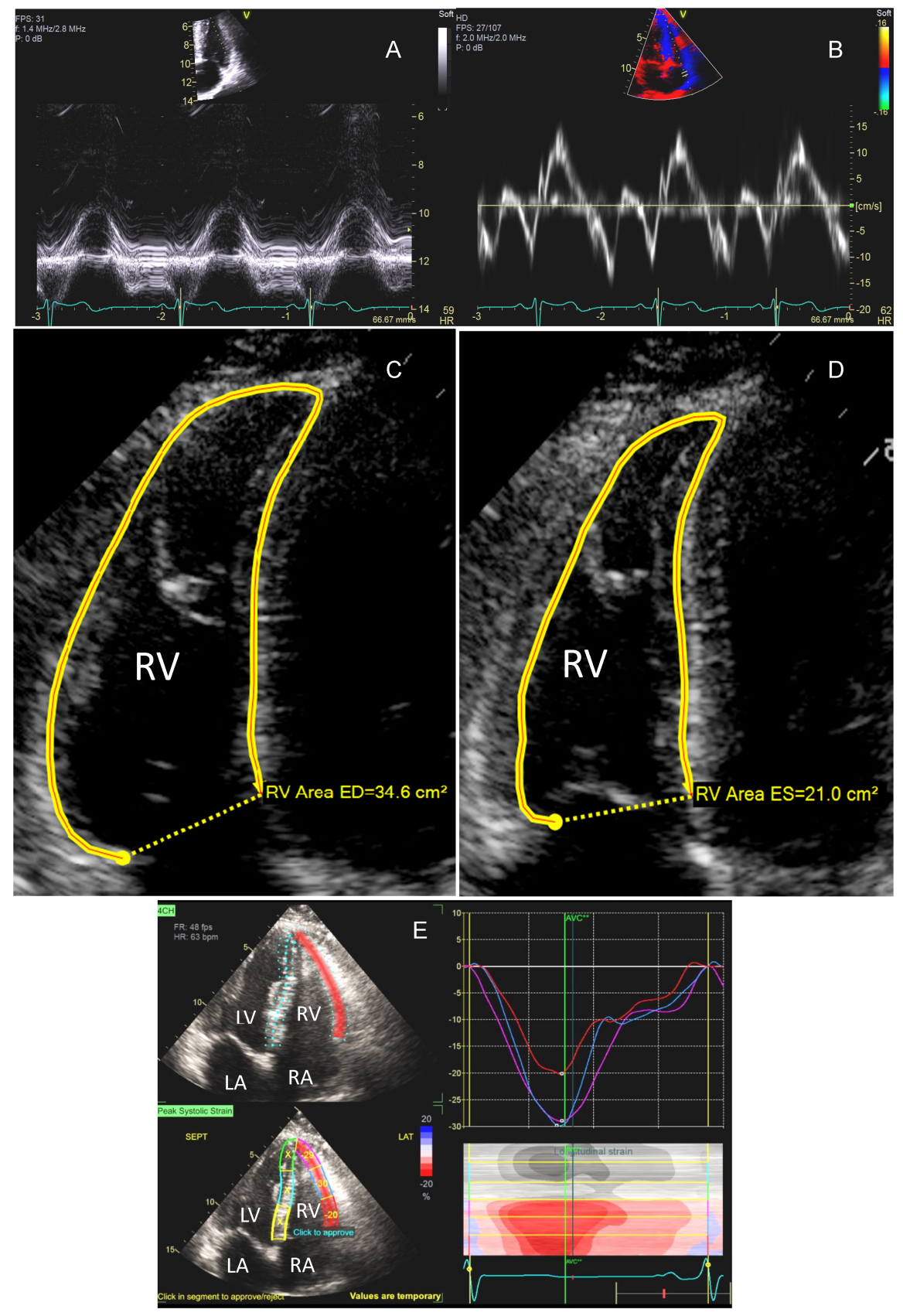



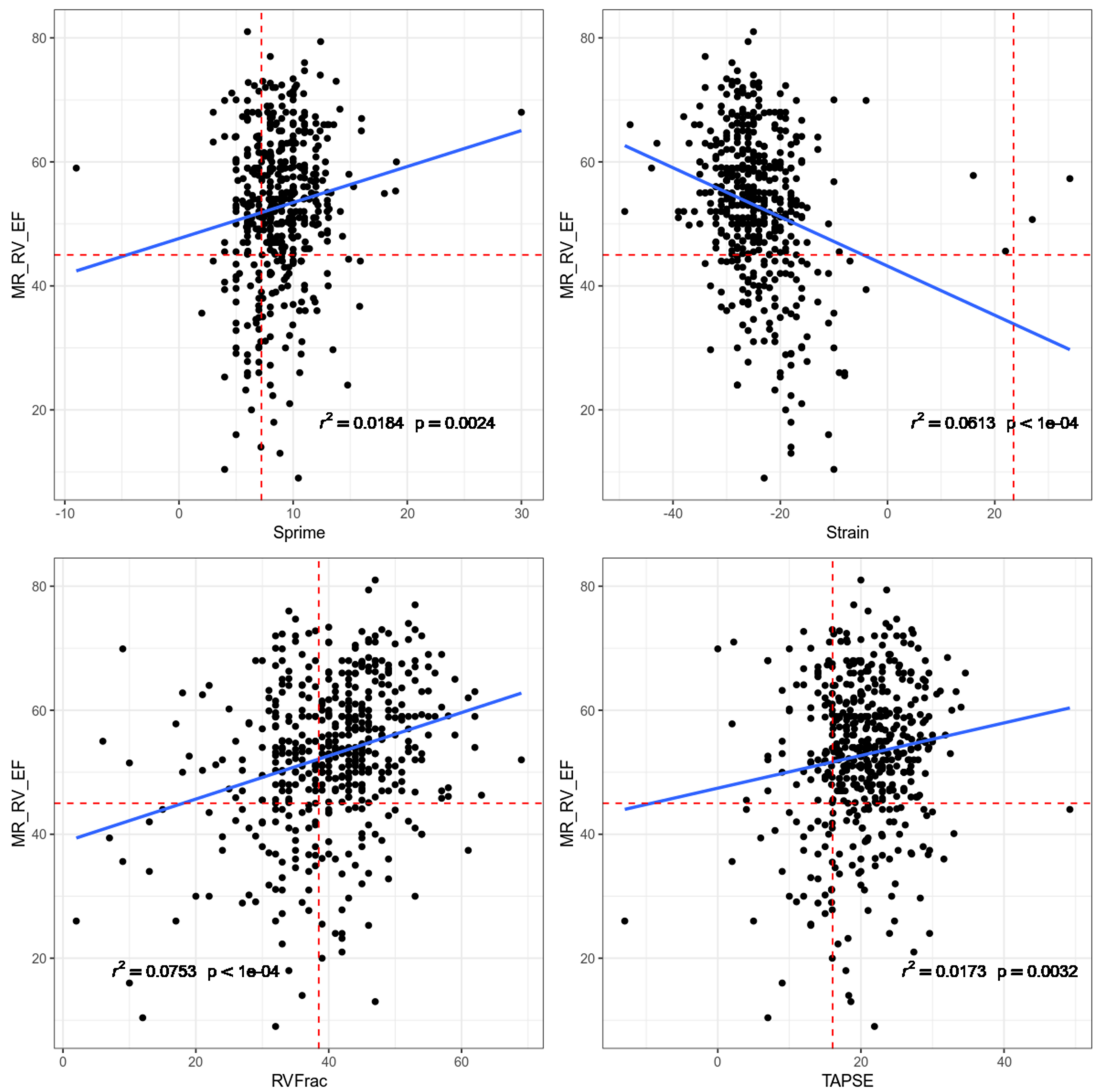

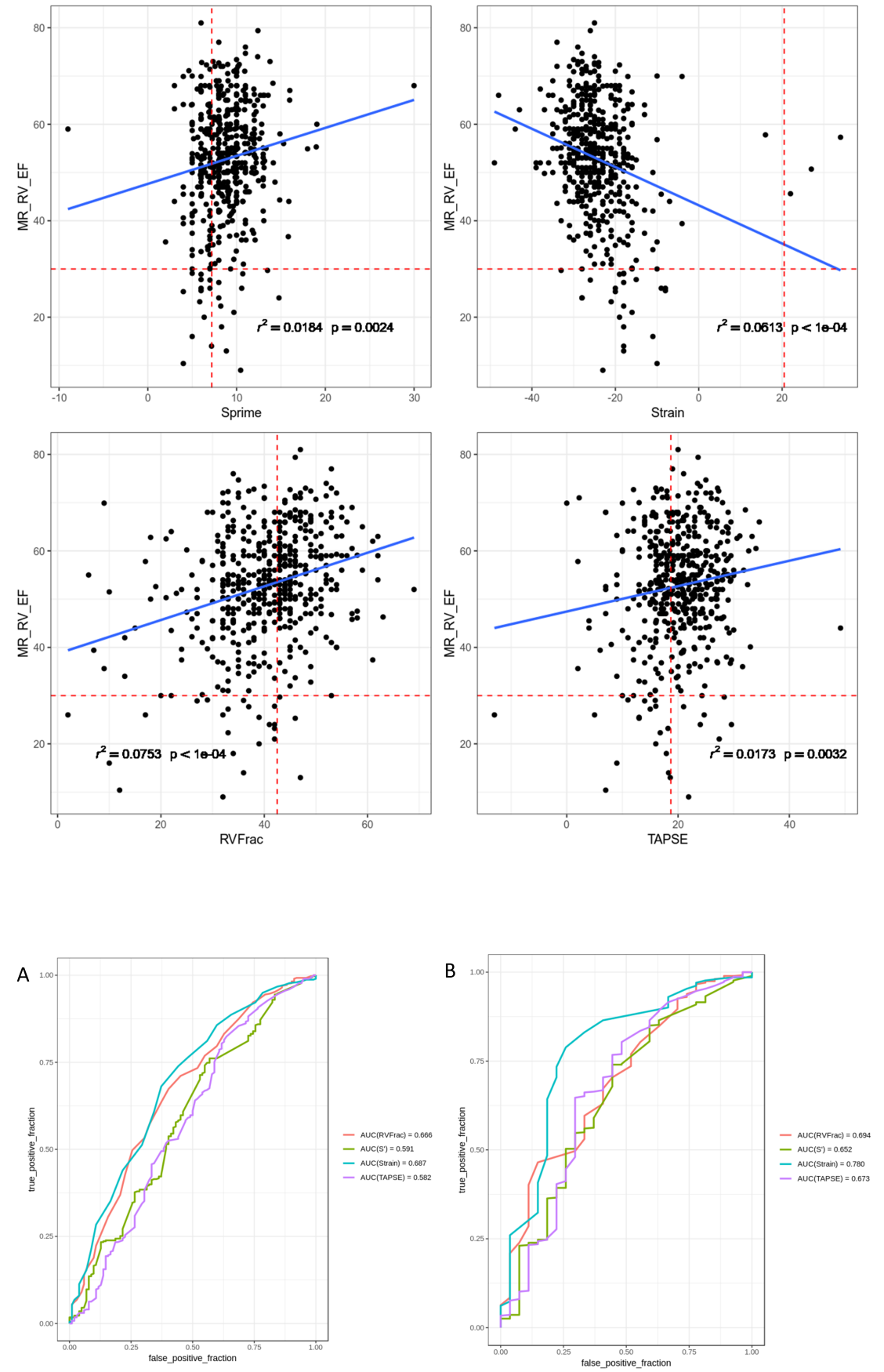